Proceedings of the lowa Academy of Science

\title{
A Practical Electrical Method of Measuring the Distance Between Parallel Conducting Planes, with Application to the Question of the Existence of Electron Atmospheres
}

\author{
F. C. Brown \\ State University of lowa
}

Copyright @1913 lowa Academy of Science, Inc.

Follow this and additional works at: https://scholarworks.uni.edu/pias

\section{Recommended Citation}

Brown, F. C. (1913) "A Practical Electrical Method of Measuring the Distance Between Parallel Conducting Planes, with Application to the Question of the Existence of Electron Atmospheres," Proceedings of the lowa Academy of Science, 20(1), 271-278.

Available at: https://scholarworks.uni.edu/pias/vol20/iss1/29

This Research is brought to you for free and open access by the lowa Academy of Science at UNI ScholarWorks. It has been accepted for inclusion in Proceedings of the lowa Academy of Science by an authorized editor of UNI ScholarWorks. For more information, please contact scholarworks@uni.edu. 
A PRACTICAL ELFCTRICAL METHOD OF MEASURING THE DISTANCES BEWTEEN PARALLEI, CONDUCTING PLANE.S, WI'TH APPLICATION TO THE QUESTION OF THE FXISTENCE OF ELECTRON ATMOSPHERES.

BY F. C. BROWN.

In the Philosophical Magazine for August, 1912, Professor R. W. Wood raises the interesting cuestion concerning the existence of conducting atmospheres surrounding metallic surfaces. The necessity for this hypothesis arose from a number of experiments, in which electrical conduction took place between metallic surfaces, when they were separated between 20 and 30 wave lengths of sodium light. The result appeals to the imagination but it is not easy to reconcile it with the well known experiments by Earhart, ${ }^{1}$ Hobbs, ${ }^{2}$ Kinsley, ${ }^{3}$ Almy, ${ }^{4}$ Williams, ${ }^{5}$ and others who found using interferometer methods that there was good insulation when the conductors were much closer than the distances observed by Wood. Perhaps the argument may be urged against the interferometer methods that no allowance was made for a possible deformation of the metallie surfaces under the electrical stresses, nor have such methods as used been infallible in detecting the absolute point of contact between the surfaces.

And Wood's experiments considered alone do not seem consistent with the electron atmosphere hypothesis, for such an atmosphere should extend out the same distance from the surface regardless of the insulating material between those surfaces, particularly so when the material is in discontinuous sheets. His results should not have shown a greater variation than the variation of his optical flat from that of a true plane.

I have tested out an electrical method for measuring small distances in order to gain information toward explaining the above discrepancies. I have found the method useful and the application interesting. The

${ }^{1}$ Phil. Mag. (6) 1, p. 147, 1901.

${ }^{2}$ Phil. Mag. (6) 10 , p. 617,1905 .

${ }^{3}$ Phil. Mag. (6) 9, p. 692, 1905.

${ }^{4}$ Phil. Mag. (6) 16, p. 156, 1908.

sphys. Rev. 31, p. 212, 1910. 
application can be most readily understood, after the method has been explained.

The electrical method of measuring the distance between two plane conductors is based on a measurement of ther electrical capacity which the two planes possessed by virtue of their nearness. For small distances such as I used the distortion of the lines of force at the edge of the planes was so small that no appreciable error accrued therefrom, and the capacity of the connecting wires and the upper plane isolated was only from three to five units and could be corrected for or neglected. This method involves the elementary but fundamental and absolute formula,

$$
\mathrm{C}=\mathrm{S} / 4 \pi \mathrm{d}
$$

where $\mathrm{C}$ is the electrical capacity of the two neighboring surfaces of area $\mathrm{S}$, and, $\mathrm{d}$, is the distance between the electrical charges on opposte planes.

Now if it should be revealed that the distance between the opposite electrical charges is the same as the distance between the mechanical surfaces, then obviously it should be concluded that within the accuracy of measurement the electrical and mechanical surfaces are identical. In other words the identity would establish that an electron atmosphere does not extend beyond the mechanical surface. The question as to whether both negative and positive atmospheres exist need not be considered at this time.

The capacity of two parallel plates can be measured readily to a satisfactory degree of precision by the method of mixtures. I used a Dolazaleck electrometer of 20 e.s.u. capacity for comparison capacity and also for measuring the necessary potentials. The procedure was first to charge up one pair of quadrants to a potential $V_{1}$, represented by a deflection of the needle, $D_{1}$. The quantity of electricity on the needle was then allowed to distribute itself between the electrometer of capacity $\mathrm{Ce}$, and the parallel plates of capacity $\mathrm{C}$. The resulting potential $\mathrm{V}_{2}$, gave a deflection $\mathrm{D}_{2}$. As usual the capacity of the parallel surfaces is,

$$
\mathrm{C}=\mathrm{Ce}\left(\mathrm{D}_{1}-\mathrm{D}_{2}\right)
$$

$\mathrm{D}_{2}$

Substituting the value of the capacity in equation (2), the distance between the conducting surfaces is obtained in the form

$$
\text { d. S. } \mathrm{D}_{2} / 4 \pi \mathrm{Ce}\left(\mathrm{D}_{1}-\mathrm{D}_{2}\right)
$$


Because of the low capacities to be compared this method requires a high degree of insulation for all the parts. But I have been very much surprised to find how easy it is to obtain almost perfect insulation when the neighboring plates are separated by only a few wave lengths of light. The only precaution thus far found is that the insulating material should bee free from moisture and impurities. In general if material once dried out is kept a few degrees above the atmospheric temperature, trouble due to moisture will be avoided. But for very thin insulating material hours of heating may be required, so that it may insulate properly the conductors which it separates.

In these experiments I measured the distances between planes when the resistance was of the order of $10^{12}$ olhms, whereas Wood measured the distances when the conductivity could be measured by a galvanometer and a milli-ammeter. Naturally then I should have expected to work with planes separated farther than 20 or 30 wave lengths, but I was a little surprised to find almost complete insulation at one or two wave lengths, when: the precautions mentioned were taken.

In 1908 (Phil. Mag. (6) 18, p. 649) I obtained insulating films which were only about $10^{-6} \mathrm{~cm}$. thick. This is nearly a hundred times less than certain minimum insulating distances obtained by Wood. One of Wood's experiments indicated that a coating of oil did not interfere with the conductivity. So I tried first a film of turpentine. I evaporated a film of turpentine at high vacuum and allowed it to condense on a highly polished brass surface. After condensation I was able to bring up in vacuum a globule of mercury from below. The globule of mercury when earthed and brought up to the film altered the capacity of the electrometer system by the amount of the condenser formed. I was able to obtain by this method insulating distances of about $6 \times 10^{-5} \mathrm{~cm}$. As I was unable to measure the film thickness by a check method, it was not safe to conclude that the electron atmosphere did not extend into the turpentine.

The first experiments were made with conducting planes of hardened steel. They were made and polished by our instrument maker, Mr. M. H. Teeuwen, but the surfaces were not tested at the time optically for planeness, and the miscroscope showed them not to be free from scratches. However at the conchusion of all my experiments they were tested and found to vary from a plane by less than two wave lengths. The area was about 1.8 sq.em.

The two planes were separated by sulphur particles collected from an air suspension. Somewhat more than a thousand particles covered the 18 
Proceedings of the lowa Academy of Science, Vol. 20 [1913], No. 1, Art. 29 274 IOW A ACADEMY OF SCIENCE

surface of one of the plates, of which about 20 were larger than $0.007 \mathrm{~cm}$. as seen by the microscope. When one of the plates was laid on the other there was complete insulation, i.e. when the condenser was charged the potential did not vary noticeably in five minutes. The observations are shown in the following table:

\begin{tabular}{|c|c|c|c|c|}
\hline $\begin{array}{l}\text { Approximate potential across sul } \\
\text { phur particles. Volts } \ldots \ldots \ldots \ldots\end{array}$ & 4.3 .4 & 3.4 & 1.8 & 0.6 \\
\hline Zero reading of electrometer, $D_{1}$. & 186 & 186 & 186 & 186 \\
\hline $\begin{array}{l}\text { Reading of electrometer when } \\
\text { charges are mixed, } D_{1} \ldots \ldots\end{array}$ & & $286,289,286$, & 245,2 & $205,201,201$ \\
\hline $\begin{array}{l}\text { Reading of electrometer when } \\
\text { charges are mixed, } D^{2} \ldots \ldots \ldots\end{array}$ & $228,237,234$, & $225,223,226$, & 205,206 , & $192,193,192$ \\
\hline 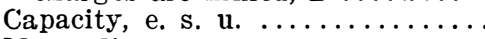 & 36 & 32 & 40 & 28 \\
\hline
\end{tabular}

These results indicate that the electrical method gives a shorter distance than the diameter of the sulphur particles, but it was not certain if the few largest surplus particles might not crush or give or roll into the scratches under the pressure of the upper plate. Further it was not clear what action so many small particles would have on the dielectrict constant of the intervening space.

Because the sulphur particles were not uniform in size and possibly not rigid, together with the difficulty of ridding of the moisture, I decided to try quartz fibres. A small fibre was broken into two parts and placed between the two surfaces. It was a day in early May when the humidity was low, and the fibres were not previously heated, except in the oxygen flame when blown. Nevertheless the insulation was regarded as perfect. The following are the observations with these quartz threads separating:

Approximate potential between plates, Volts 2

$\mathrm{D}_{1} \ldots \ldots \ldots \ldots \ldots \ldots \ldots \ldots \ldots \ldots \ldots \ldots \ldots \ldots \ldots \ldots \ldots \ldots, 319,317$,

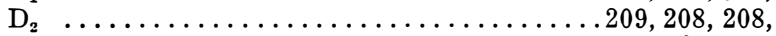

Capacity ..................... 100

Corrected capacity ................ 97

Mean distance
0.9

$242, \quad 245,245,244,245$

$195.5,196,195,197,195$

103

100

Now the size of the quartz fibres separating the planes as determined by the micrometer evepiece on the microscope was

$$
.00165 \pm .000086 \mathrm{~cm} \text {. }
$$

This difference between the distance then varies by only about two wave lengths. But $I$ found later in reading the size of fine fibres by the microscope, that it was much easier to err by obtaining values too large than too small. This experiment then leads to the conclusion that if there be a conducting atmosphere, that it must not extend out more than two wave lengths. 
Obviously the conclusion just arrived at is inconsistent with Prof. Wood's experiment where he obtained a current of several milli-amperes when parallel planes were only five wave lengths apart. I therefore thought it advisable to repeat the experiment using smaller quartz fibres, this time three in number. The sizes were, first, $.00052 \mathrm{~cm}:$ see.nnd, .00053 ; third, .00059 and the mean probable distance of separation was therefore $.00056 \mathrm{~cm}$. The electrical method by the mean of five observations, with 0.3 volt across condenser, gave a separating distance of $.00054 \mathrm{~cm}$.

There are two conclusions that should be drawn from this experiment. First there was almost perfect insulation when the two planes were separated by only ten wave lengths and second the conducting atmosphere could not have extended as much as two wave lengths outside the mechanical surface.

A further attempt to repeat the above experiments with yet finer quartz fibres met with failure owing to unsatisfactory insulation. I did not try heating the quartz fibres at this time because I feared that it was steel surfaces might vary by ten wave lengths. I observed that it was impossible by the electrical method to obtain insulation at distances less than those measured by the microscope. Unless some unknown factor is involved this observation obviously leads to a third conclusion, which is that conduction at short distances arises from conducting material and not from an electron atmosphere of unvarying depth.

The question as to the imperfect insulation at less than 9 wave lengths was still unanswered. Possibly the insulating properties of certain dielectrics break down when a certain minimum thinness is reached. It was thought that a constant minimum thinness for any given material would strengthen this view. Or perhaps as Wood suggested moisture films about small particles might readily explain the imperfect insulation if not also the high conduction. We shall recur to this point in a succeeding paragraph. But the most opportune explanation, whose plausibility should be considered, was the possible irregularity of the steel surfaces.

The question of the planeness was answered in two ways. First a glass plate with plane sides was moved over the surface of the plates, and the character of the interference fringes noted. By this test the extreme variations of the surface was not as much as two wave-lengths.

Second two optical flats on speculum metal were obtained, whose surfaces by optional test did not vary from a plane as much as one wave length. With these my surprise was certainly great when I found imperfect insulation when the surfaces were separated by quartz fibres of " 24 wave lengths diameter. As my supply of quartz fibres, and also of 
oxygen was exhausted, I now turned to the use of mica flakes for insulation material. First when a continuous sheet of mica of 25 wave lengths separated the flats the insulation was imperfect. If charged to a potential of 4 volts, three-fourths of the charge would leak off in 10 seconds, but by thoroughly heating the plates and the mica over an electric oven the insulation became quite perfect. This was more than a month after my experiments with the iron surfaces, and I now found it quite impossible to obtain good insulation without heating the plates and the insulator. Presumably the excess humidity was the trouble.

In one instance the speculum surfaces were separated by three mica flakes of total area about $5 \mathrm{~mm}^{2}$. By the microscope one was about 14 wave lengths, the second 12 , and the third was lost. When the electrometer was charged to +5 volts and mixed with the speculum condenser the capacity was $310 \mathrm{~cm}$, and when it was charged to -5 volts the eapacity was $315 \mathrm{~cm}$. The total pressure on the upper plate was about 50 gms. From this capacity was subtracted 59 units as a correction due to the added capacity duc to the mica flakes and to the plate aside from its condenser action. The area of the plates was $1.84 \mathrm{~cm}^{2}$. The distance between the surfaces was therefore calculated to be $.00060 \mathrm{~cm}$. It seems safe to conclude from this experiment that the difference between the electrical and optical methods is not more than three wave lengths.

It was observed that if the pressure on the plates was increased to $100 \mathrm{gms}$. that the insulation became very poor. Sometimes the insulation would be restored by removal of the pressure and sometimes not. However, increased pressure did not always alter or destroy the insulation.

Again three very thin mica flakes were selected. By dircet reflection they all appeared a brilliant green, hut when viewed by the light that struck at a very large angle of incidence some of them appeared orange colored. With these fakes between the flats the speculum slid over each other freely as if nothing was between, and likewise they resisted pulling apart. The resistance between the plates was very low, although I did not measure it. Continued heating with the two plates together did not restore the insulation, although the mica had been heated for hours before the flakes were separated off the sheet mica.

Then the plates were separated and heated for two hours over an electric oven. The temperature of the plates was kept at about $215^{\circ}$. The mica flakes stuck tight to the plates during this procedure, although some of them did break in pieces. By this the insulation was made 
almost satisfactory, as may be seen from the following observations. It was necessary to determine the deflection from the first few swings of the needle. The period of the electrometer needle was about 10 sec.

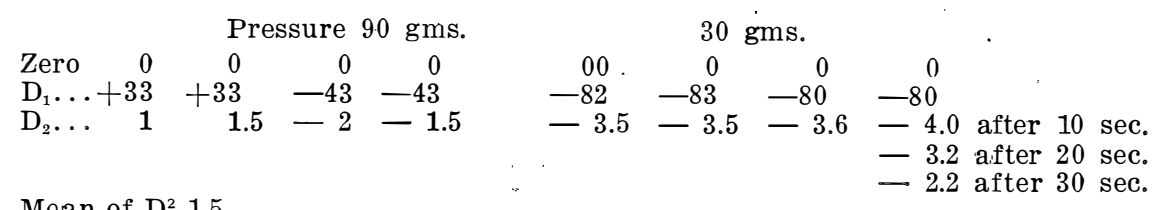

Mean of $\mathrm{D}^{2} 1.5$

Mean .............3.65

Corrected mean ....4.45

Distance

$.00031 \mathrm{~cm}$.

$.00043 \mathrm{~cm}$.

Since heating the mica and plates to $215^{\circ}$ had made the insulation so nearly satisfactory, it seemed wise to repeat this treatment. The plates were kept covered with japanese silk tissue to prevent dust particles from settling on the plates. After six hours the plates were removed while hot and tested at once. The insulation was not perfect but it was improved and at the same time the capacity was: larger. At the beginning of the test the natural leak was such that the electrometer needle went from 3 div. to 1 div. in 50 sec. At the end of the observations the natural leak was from 2.7 to 1 in 20 seconds, from 1.0 to .5 in 30 sec., showing that the insulation deteriorated with time, possibly owing to the formation of a moisture film as the plates cooled. The natural leak was corrected for in calculating the distance. The following observations were obtained before the insulation deteriorated seriously:

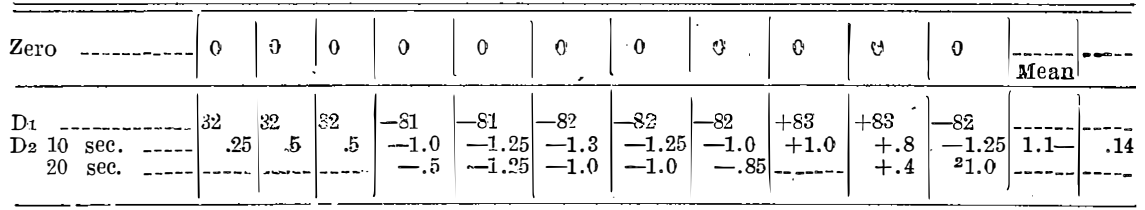

Mean corrected 1.4 .

Capacity 1170.

Distance, em., .00013.

I was not aible by the microscopic method to determine the thickness of the mica flakes, further than to limit their probable size to less than five wave lengths. This much also was determined by a micrometer screw gauge. Then interference fringes were formed by bringing two optical flats on glass into contact along one edge and allowing the opposite edges to be separated by the mica flake. The number of firinges between the point of contact and the mica obviously gives twice the thickness of the mica in wave lengths. However, the great difficulty in this measurement was that sometimes considerable pressure had to be 
applied in order to bring the plates into contact at one edge, much greater than was applied to the speculum metal plates. However, there was considerable irregularity in the pressure required to cause contact, and it may have been that after heating the speculum metal plates were separated only by the thickness of the mica. At any rate one of the mica flakes measured two and a half wave lengths, another one two, and a third one only one wave length.

I observed after the second heating that the mica flakes on speculum surfaces showed the same deep green color that they exhibited when free. This rather indicates close contact between the mica and the speculum. Also the fact that long continued heating permitted the electrical charges to become closer together, when separated by mica, indicates that heating either drives off something that conducts and separates, or alters the structure of the surfaces. If the mica flakes were in contact with the speculum, then there can be no conducting atmosphere of more than two wave lengths of light, for this I regard as about the safe accuracy of my experiments, but if on the other hand there exists a conducting atmosphere which is at the same time a mechanically separating medium, then it would not be safe to set such a low limit on the thickness of a possible electron atmosphere. The question will not be definitely settled until the distances are measured simultaneously by the two methods. We propose to do this by using very thin films of platinum deposited on plane glass surfaces. It is hoped thereby to finally decide to a fraction of a wave length whether or not an electron atmosphere exists, and also to obtain information on the role played by insulating materials of small dimensions.

Inasmuch as heating certain insulators such as mica and quartz restored their insulating properties, it is certain that the conduction obtained by Wood at such long distances was due to the presence of the insulating material, and it is presumed that moisture films and conducting particles were responsible for it.

In conclusion it may be said that while the electrical method was satisfactory as here used, it can be more accurately adapted to the problem in hand by more suitably choosing the suspension for the electrometer needle and by using a larger capacity in parallel with the electrometer.

The State University of Iowa. 